\title{
Patient's Unique Identifier for Efficient and Secure Monitoring of Pregnant Women in Burkina Faso
}

\author{
Seydou Golo BARRO ${ }^{\mathrm{a}, \mathrm{b}, 1}$, Adrien $\mathrm{UGON}^{\mathrm{c}, \mathrm{d}}$, Nadège R. NANA ${ }^{\mathrm{a}}$ and Pascal STACCINI ${ }^{\mathrm{c}}$ \\ ${ }^{a}$ Nazi BONI University, Bobo-Dioulasso, Burkina Faso \\ ${ }^{b}$ RETINES Lab - Cote d'Azur University, Nice, France \\ ${ }^{c}$ ESIEE, Paris, France \\ ${ }^{d}$ Sorbonne Université, CNRS, LIP6, F-75005 Paris, France
}

\begin{abstract}
The objective of this work is to set up a device allowing to identify the pregnant woman in a univocal and reliable way during her pregnancy follow-up. This study is a continuation of a project to improve the electronic monitoring of pregnancy in pregnant women in Burkina Faso. The methodology is based on the scientific work of the GMSIH of France (1). The work has lead to the design and implementation of a model that allows to assign a "Unique Identifier" to each pregnant woman from her first prenatal visit. The Patient ID is developed from the person's identification trait profile. It consists of a sequence of 20 characters and a security "key" of 2 characters. After the design, a reliability test of the model was performed to take into account identity anomalies (duplicates, collisions).
\end{abstract}

Keywords: Unique Identifier, Patient Identification System, pregnancy monitoring

\section{Introduction}

Pregnancy monitoring is a major problem in Burkina Faso but also in most developing countries. With the development of digital technology and particularly of "communicating computing", it is now possible to envisage a dematerialization of the exchange of information on the patient. Under these conditions, the follow-up of the patient or the pregnant woman requires fluid access to information wherever it may be and especially without getting the wrong person. However, the diversity of identification systems within healthcare systems entails increased risks of errors with enormous and sometimes fatal consequences. In the US, a collective of 27 organizations led by the American Health Information Management Association (AHIMA) published an open letter to representatives on June 11,2019, reporting that misidentifications cost US \$ 17.4 million $(15.5 \mathrm{M} €)$ per year on average at a healthcare center, due to loss of income and refused reimbursement requests (2). This is what justifies the need for a reliable identification system in any healthcare system, which is an essential condition for secure information exchanges. Identifying the patient in the information system consists of assigning him a new identifier or finding an existing identifier, from his external characteristics called identification "traits", supposed to represent a person in order to identify him uniquely in this. system. There are three types of traits: strict traits, extended traits, complementary traits. These three types of traits are distinguished according to their more or less easy to obtain, more or less stable, more or less confidential and more

1 Corresponding author, Seydou Golo Barro, Nazi BONI University, Bobo Dioulasso; E-mail: seydou.golo@gmail.com. 
or less discriminating character. The unique identifier is a sequence of numeric or alphanumeric characters. It is the "key" to access information concerning the person admitted to the care system. Even in developed countries, even today, the electronic exchange of health data raises many debates concerning patient privacy, the choice of patient identifier and associated technical devices (3).

Problematic of the unique patient identifier: The issue of the unique patient identifier is raised in most countries around the world and each country has its own identification process. Patient identification is an important part of providing care and is the first step in a process that lasts throughout the care journey. It is one of the important parts in the health information system and contributes to the evolution of the health care system. A study report by the Group for the Modernization of the Hospital Information System (GMSIH), "Principles and process of patient identification": International experiences of health systems and identification policy", presents the experience of some countries in patient identification. The report classifies countries into three categories (4).

- The countries where the national unique identification of the patient is organized or planned: countries of the North of Europe (Denmark, the Netherlands, Belgium, Great Britain, Ireland) and New Zealand, Germany?

- Countries where the patient identification is more of the order of the "region": Southern European countries (Spain and Italy), federal countries (Canada and Australia).

- The recent undecided countries: the United States of America, which is moving towards the federation of identifiers.

In Africa, for many health facilities, the precise identification of patients remains a challenge. This is due to the insufficient quality of information systems and the absence of national systems for the identification of persons (5) (6). In Burkina Faso, the unique patient identification system is not yet used. This raises the issue of the security of information exchange and the continuity of care when the patient returns to the system for further care. Initiatives are underway for the establishment of a unique identification system for Burkinabé citizens and a unique national identifier. However, none of these initiatives have yet come to fruition. It is in the light of all these issues that we decided to design a unique patient identification model in a Burkinabé context. As part of this work, the unique identifier model is being tested in the monitoring of pregnancy and childbirth.

\section{Methods}

The method used for the design and implementation of the patient ID system is based on the scientific work of the GMSIH of France.

\subsection{Creation of the Burkinabè model of unique identifier (patient ID)}

According to the work of the GMSIH, a unique identifier of quality must be built with reliable features, always available. These traits must allow a bijective relationship between the physical person and his identity. There are three types of identifying traits: Strict traits, extended traits, complementary traits. These three types of traits are distinguished according to their more or less easy to obtain, more or less stable, more or less confidential and more or less discriminating character. Some traits are more stable than others. These are retained to constitute the "strict features": generally the names, 
first names, date of birth and sex. Traits that are more unstable over a person's life, such as marital status or address, form the "extended traits". They serve as discriminating elements rather of an administrative nature, when the strict features are insufficient to remove any doubt about an identity. Finally, "complementary traits" are health information, which allows healthcare professionals to discriminate against people even more finely. In our case, we have retained the following traits: "Strict traits": family name, first name (s), date of birth, sex. "Extended features": place of birth. "Complementary features": Address of residence.

\subsection{Criteria for creating the patient ID}

To achieve a valid identification it is necessary to respect a certain number of rules: We have therefore developed an identification protocol to create a unique identifier in Burkina Faso taking into account the local context. It breaks down into four stages:

1) Use of identification traits. It consists in recovering the strict and secondary traits of the patient necessary for the creation of the unique identifier.

2) Standardization of characters. This step allows you to format the identification traits. It is:

- Standardize the name and first name fields in an appropriate format (upper case, no accents, no spaces for compound names);

- Perform a first hash (using the sha512 hash algorithm) of the first and last name;

- Code the sex field in the format 1 for male and female;

- Standardize the date of birth in this format: ddmmyyyy ( 8 digits);

- Retrieve the code of the place of birth for the "place of birth" field. If the persons were born abroad the place of birth code is 00099 ;

$\circ$ Retrieve the city of residence code for the residence address field. If the persons reside abroad, the residence code is 00099;

- Carry out a concatenation on the result of the preceding operations according to the following format: (sex_code.ddmmyyyy.hash (namefirstname) .birth_ocalitecode.codelocalite_residence.sector_num);

○ Keep only the digits of this concatenation and convert the letters into their corresponding in-tier according to the ASCII encoding.

3) Hash: perform a second hash of the result obtained with the "sha512" algorithm, the goal being to strengthen the security of the identification system. After this concatenation, take the first 20 characters from the index 50.

4) Calculation of the control key: The control key ensures the validity of the identifier. It is determined from the constituent characters of the identifying code. By performing a Euclidean division by 97 of the numbers resulting from the hash, we obtain a remainder which will be subtracted from 97 . The result of this subtraction is the control key.

\section{Results}

Implementation of the patient ID system: For the implementation of the system, two types of libraries "hashlib" and "unicode" which respectively allow hash function and character normalization. The intermediate functions are "Normalize (string)" which uses the unicode library with the unicodeda-ta.normalize and unicodedata.category functions. this function returns the deleted string of uppercase, underscores, spaces, numbers and 
special characters; the Con-vers_ASCII function (string, L0 = ['0', '1', '2', '3', '4', '5', '6', '7', '8', '9' ]) which takes as input a character string and a L0 list of digits from 0 to 10 . It decomposes the string into a list of simple elements using .strip; it goes through this list, and converts the letters to integers using the ord () function; it returns a list concatenated with the letters of the string converted to ASCII integers. The main functions are: 1) "trait_d_information ()". It allows you to enter the identification traits of the patient. it returns a list of traits concatenating the entered identification traits: traits = [surname_of_family, first name, date_of_birth, code_sex, code_localite_birth, code_localite_residence, sector_num]. 2) "unique_identifier (traits)" which takes as input the list of previous traits, then normalizes the first and last names fields, last name and first name hash, first con-catenation of standardized fields, conversion of the letters of the hash into their integer ASCII, new hash, selection of the first 20 characters of the hash from index 50, reconversion of the letters into their ASCII in-tier and selection of the first 20 digits, to give a first identifier, calculation of the control key, concatenation the identifier and the control key. It is also this function that revolves the Unique Patient Identifier.

Integration of the unique patient identifier method in an HIS: Patient ID works within a hospital information system. We test our model by integrating it into the electronic pregnancy monitoring system of the University Hospital of Bobo-Dioulasso (e-Lafia) (7). E-Lafia is a Pregnancy Tracking Information System. Patient information is collected in e-Lafia through a registration form. This information is stored in a MySQL database. E-Lafia HIS has been selected since it uses healthcare interoperability standards such as HL7v3, FHIR, DICOM, thus facilitating interaction with our unique identification system.

\section{Conclusions}

This pilot experience of designing and implementing a unique patient identification system within a pregnancy monitoring system is an innovation and a basis for computerization and data exchange projects on the patient. Although functional, our patient ID system could be improved to integrate identity reconciliation procedures with traceability of all operations; the creation of an identity-vigilance unit which will ensure the conditions for correct data collection, the reporting of anomalies and duplicates as well as the inclusion of corrections.

\section{References}

[1] GMSIH. Identification du patient: Guide pour l'élaboration des politiques d'identification et de rapprochement: Annexe 1 : Charte d'identification. 2002.

[2] USA : vers un identifiant patient unique au niveau national [Internet]. Innovation Mutuelle. 2019 [cité 24 sept 2020]. Disponible sur: https://www.innovation-mutuelle.fr/actualite/usa-vers-un-identifiant-patientunique-au-niveau-national/

[3] Dumont V. Controverses autour de l'échange électronique de données de santé. La question de l'identifiant du patient. Termin Technol Inf Cult Société. 1 juin 2011;(108-109):125-38.

[4] GMSIH. Principes de l'identification du patient - Tome 2. Paris: GMSIH; 2002 avr p. 80. (Modele de rapprochement).

[5] Bacher SC. L'identification du patient. :106.

[6] Bediang G, Amougou EE, Essi M-J. Identification des Personnes Physiques et des Patients: Une Étude de Cas au Cameroun. 2019.

[7] Kaboré AA, Kaboré BE, Barro SG. e-Lafia: Suivi électronique de la grossesse. Université Nazi BONI; 2020. 